\title{
Quality Engineering: Designing for Quality - the SW Engineering Challenge
}

\author{
Ulf Olsson \\ (Invited speaker) \\ Bofors Electronics \\ S-175 88 Järfälla, Sweden
}

\begin{abstract}
Hardware quality can be expressed as the probability that something will break and/or wear out. Software does not wear out, but it can break when exposed to overload (i.e., unexpected or unfortunate sets of inputs, resource failure, ...). A trustworthy system will behave in a controlled way under such circumstances: it will perform as well as possible, and will in general not compound the problem. Quality Assurance (QA) aims at removing the sources of untrustworthiness (bugs, errors, features) by forcing developers to prove to someone with project authority (but possibly with only superficial technical knowledge) that enough thought and work has gone into trying to reduce those risks.

This talk is an attempt to set QA in the perspective of the developers. The basic point of the argument is that QA is a supportive function. If not controlled, there is a risk that QA (and Configuration Management) procedures are formalized and expanded so far that they actually become counter-productive: less time is spent on the product as such; i.e., quality goes down. Obviously, there is a balance to be struck here. A few points will be made from our experiences, illustrating this and showing how we try to think at least as much about the properties of the products as about those of the process! Actually, the two go hand in hand: improving the process gives better products; improving the products provides helpful hints about where the process needs further enhancement.
\end{abstract}

Ulf Olsson graduated from Stockholm's Royal Institute of Technology in 1978, with a M.Sc. in Engineering Physics. Since then, he has been with Philips Elektronikindustrier (which became Bofors Electronics in 1989). He has been involved as a Software Engineer and Software Project Manager in a number of projects, including several Coastal Artillery systems, airborne as well as land-based radar extractors, and C3 systems. Since 1985, he has been involved in the SS2000 projects in various capacities, mainly concerning overall software design. In 1989, he was appointed Senior Specialist in the field of System Architecture. 